1 Diversity in the oligodendrocyte lineage: plasticity or heterogeneity

2

3 Sarah Foerster, Myfanwy FE Hill and Robin JM Franklin

4

5 Wellcome Trust-Medical Research Council Cambridge Stem Cell Institute, Clifford Allbutt

6 Building, Cambridge Biomedical Campus, University of Cambridge, Cambridge CB2 OAH, UK

7

\title{
8 Acknowledgements
}

9

10 The authors acknowledge the support of funding from the UK Multiple Sclerosis Society,

11 Medlmmune, The Adelson Medical Research Foundation and a core support grant from the

12 Wellcome Trust and MRC to the Wellcome Trust-Medical Research Council Cambridge Stem

13 Cell Institute. SF and MFEH have been recipients of Wellcome Trust funded PhD studentships.

14

15 Word count

16

17 Total: 7139

18 Abstract: 172

19 Introduction: 193

20 Main: 3504

21 Concluding remarks: 530

22 
Abstract

Heterogeneity is a widely recognised phenomenon within the majority of cell types in the body including cells of the central nervous system (CNS). The heterogeneity of neurons based on their distinct transmission modes and firing patterns has been recognised for decades, and is necessary to coordinate the immense variety of functions of the CNS. More recently, heterogeneity in glial cells has been described, including heterogeneity in oligodendrocyte progenitor cells (OPCs) and oligodendrocytes. OPC subpopulations have been described based on their developmental origin, anatomical location in the grey or white matter, and expression of surface receptors. Oligodendrocytes are categorised according to differences in gene expression, myelinogenic potential and axon specificity. Much of what is described as heterogeneity in oligodendrocyte lineage cells (OLCs) is based on phenotypic differences. However, without evidence for functional differences between putative subgroups of oligodendrocyte lineage cells (OLCs), distinguishing heterogeneity from plasticity and lineage state is difficult. Identifying functional differences between phenotypically distinct groups is therefore necessary for a deeper understanding of the role of OLCs in health and disease.

\section{Key words}

oligodendrocyte, oligodendrocyte progenitor cell, heterogeneity, myelin, remyelination

\section{Main points}

1. Phenotypic differences have been described between subpopulations within the cells of the oligodendrocyte lineage.

2. Heterogeneity cannot be distinguished from functional plasticity based solely on 50 phenotypic differences.

3. Distinct functional differences between subclasses of oligodendrocyte lineage cells need to be demonstrated unambiguously to prove heterogeneity. 


\section{Introduction}

The central nervous system (CNS) integrates information it receives from all parts of the body, and in turn coordinates and influences their activity. To coordinate this immense variety of functions, different neuronal subtypes with distinct transmission modes and firing patterns are necessary. Similarly, region-specific astrocyte functions are required for the maintenance of CNS homeostasis and neuronal survival (Tsai et al., 2012). These examples demonstrate a functional heterogeneity of different cell types in the CNS, raising the question whether a similar heterogeneity exists for oligodendrocyte lineage cells (OLCs, an umbrella term for oligodendrocyte progenitor cells (OPCs) and their progeny oligodendrocytes). Evidence for diversity within both the oligodendrocyte and OPC populations has accumulated over the last decade. However, there is not yet a fully coherent perspective on the functional implications of this diversity or the extent to which this diversity represents true heterogeneity as distinct from functional plasticity. There are several different methods of categorising heterogeneity of OPCs and oligodendrocytes, many of which are not mutually exclusive. Here we examine the evidence in support of the OLCs being a heterogenous cell population and discuss what the functional roles for these different sub populations might be.

\section{Definition of heterogeneity}

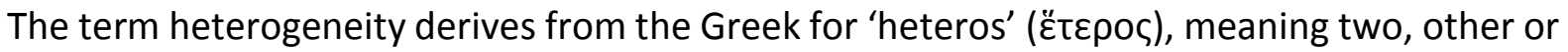
different, and 'genesis' from the Latin, originally borrowed from the Greek ( $ү \varepsilon ́ v \varepsilon \sigma \iota \varsigma)$, meaning origin or development (Oxford English Dictionary). Therefore, implicit in the term is the sense that, for a population to exhibit heterogeneity, its components must have distinct developmental origins. However, currently it is more commonly used to describe a situation where, in addition to origin, a single cell type can show distinct morphological and/or phenotypic profiles, including gene expression, and a distinctive range of functions including proliferation potential, motility, and response to injury. The gold-standard to unambiguously identify heterogeneous populations of a cell type is the proof of functional differences. A critical point is that true heterogeneity should not be confused with identification of cells at different cell states within a cell population (e.g. adult versus adult activated OPCs following 
injury), which is better termed functional plasticity, or cells captured at different points along a differentiation path (e.g. pre-myelinating versus mature oligodendrocytes).

\section{Defining OPCs and Oligodendrocytes}

In the adult CNS, OPCs are estimated to comprise at least $5 \%$ of all cells, residing in both white and grey matter (Dawson, Polito, Levine, \& Reynolds, 2003; Pringle, Mudhar, Collarini, \& Richardson, 1992). Typically, OPCs are identified by the presence of the proteoglycan NG2 (Stallcup \& Beasley, 1987) or by platelet derived growth factor receptor A (PDGFRA) (Pringle et al., 1992). In vivo lineage tracing studies show that the vast majority of OPCs express both NG2 and PDGFRA (Figure 1) (Kang, Fukaya, Yang, Rothstein, \& Bergles, 2010; Karram et al., 2008; Rivers et al., 2008); hence, the two marker proteins can be used interchangeably, rendering it possible to compare studies performed using either marker. Additionally, the ganglioside antibody A2B5 is used for the identification of OPCs in in vitro studies (Raff, Miller, \& Noble, 1983). Immunostaining of OPCs isolated using A2B5 indicates that the vast majority of these cells also express NG2 and PDGFRA (Figure 1) (unpublished data from our laboratory). However, neither marker is exclusively restricted to OPCs: NG2 can label activated microglia and pericytes, PDGFRA can also label pericytes while the A2B5 antibody can label neural stem cells and neurons. Therefore, to unambiguously identify an OPC, a combination of the OPC markers or co-localisation with an OLC marker, such as the transcription factors Olig2 (Zhou, Wang, \& Anderson, 2000) or Sox10 (Kuhlbrodt, Herbarth, Sock, Hermans-Borgmeyer, \& Wegner, 1998), should be used. However, as the OLC markers are also expressed by cells in later stages of differentiation they cannot alone be used for the identification of OPCs.

As an OPC starts to differentiate, marker proteins such as the ectonucleotide pyrophosphatase/phosphodiesterase 6 (ENPP6) (Xiao et al., 2016), O4 (Sommer \& Schachner, 1981) and 2',3'-cyclic-nucleotide 3'-phosphodiesterase (CNPase) (Poduslo \& Norton, 1972) are expressed, identifying a differentiation state between a progenitor and a fully mature oligodendrocyte. These pre-myelinating oligodendrocytes differentiate into cells with progressively more complex process networks and eventually mature myelin sheaths, thus becoming a mature oligodendrocyte. Mature, sheath forming, oligodendrocytes express myelin sheath proteins including myelin basic protein (MBP) (Sternberger, Itoyama, Kies, \& 
118 Webster, 1978), myelin/oligodendrocyte glycoprotein (MOG) (Linnington, Webb, \& 119 Woodhams, 1984), myelin-associated glycoprotein (MAG) (Sternberger, Quarles, Itoyama, \& 120 Webster, 1979), myelin regulatory factor (MYRF) (Cahoy et al., 2008) and proteolipid protein 121 (PLP) (Sobel, Greer, Isaac, Fondren, \& Lees, 1994).

Progression along a differentiation and maturation pathway is a continuous and seamless process. Combinations of marker proteins, all of which appear and disappear within defined phases of differentiation, can be used to define distinct stages of development, which are useful as reference points but should not be taken to imply that differentiation necessarily proceeds in quantal steps. Additionally, it should be noted that the expression of marker proteins (so far only shown for OPCs) can change with the activation state (Moyon et al., 2015), during development (Clarke et al., 2012; Karram et al., 2008; Ligon et al., 2006; Stallcup \& Beasley, 1987) (Figure 1) and/or ageing (unpublished data from our laboratory). Therefore, proof of heterogeneity inferred from marker protein expression is difficult as it may only represent lineage stage.

\section{Developmental OPC heterogeneity - does origin matter?}

During embryonic development of the CNS, OPCs are generated from radial glia cells in multiple localised areas. The diversity of OPCs based on their origin is known as developmental heterogeneity. In the murine spinal cord, most OPCs arise from the pMN domain of the ventral ventricular zone, and subsequently populating the entire neural tube (Fogarty, Richardson, \& Kessaris, 2005; Pringle \& Richardson, 1993). Additionally, a minority of OPCs is generated from progenitors in the dorsal dP3, dP4, dP5 and dP6 domains beginning at E16.5 (Cai et al., 2005; Fogarty et al., 2005; Vallstedt, Klos, \& Ericson, 2005). In the adult mouse, OPCs from ventral and dorsal regions are intermixed, with a heavy predominance of pMN-derived (ventral) cells (85-90\%). OPCs arising from dorsal progenitors mostly populate the dorsal and lateral funiculus (Tripathi et al., 2011).

Developmental heterogeneity of OPCs also occurs in the telencephalon, where OPCs arise from three distinct regions in a spatiotemporal manner. The earliest OPCs develop from the medial ganglionic eminence (MGE) and the anterior entopeduncular (AEP) region in the 
ventral developing telencephalon, starting from E11.5. Subsequently, at E16.5, a second population of OPCs are formed from the ventral lateral and caudal ganglionic eminence (LGE, CGE). Both OPC populations spread from ventral to dorsal, eventually populating the entire telencephalon. After birth, a third population of OPCs arises in the developing cortex, which populate the dorsal parts of the telencephalon (Kessaris et al., 2006). During postnatal development, the majority of the first population of OPCs from the MGE-AEP region is eliminated, leaving the adult brain with OPCs derived from the ventral LGE-CGE region and the dorsal cortex (Kessaris et al., 2006). In the adult telencephalon, dorsally derived OLCs mainly populate the cortex ( $\sim 50 \%$ dorsal OLCs, $\sim 35 \%$ ventral OLCs) and the corpus callosum (CC) ( $25 \%$ dorsal OLCs, $15 \%$ ventral OLCs), whereas the anterior commissure (AC), the preoptic tract (POA) and the lateral olfactory tract (LOT) are almost exclusively populated by ventral OLCs (Tripathi et al., 2011). The question arises, why should there be developmental heterogeneity in the oligodendrocyte lineage? Do different OLC populations fulfil different roles, or is developmental diversity simply an evolutionary ploy to accommodate for the rapid growth of the CNS?

Different molecular cues are needed for ventral and dorsal OPC specification in development. Shh-signalling is required to generate ventral OPCs but is redundant for dorsal OPC specification (Cai et al., 2005; Fogarty et al., 2005). In contrast, the induction of FGF signalling as well as the inhibition of WNT and BMP signalling pathways may play an important role in the specification and timing of appearance of dorsal OPCs (Chandran et al., 2003; Fogarty et al., 2005; Langseth et al., 2010; Vallstedt et al., 2005). In addition to differences in specification factors, dorsally derived OPCs also exhibit a preference to myelinate dorsal areas in the CNS (Kessaris et al., 2006; Tripathi et al., 2011). In the course of spinal cord development, the dorsal funiculus is initially populated by ventrally-derived oligodendrocytes but by adulthood comprises more than $80 \%$ of dorsally-derived oligodendrocytes. That ventrally derived oligodendrocyte numbers decrease after postnatal day 13 (P13), whereas dorsally derived oligodendrocyte numbers stay constant, argues strongly for a selective advantage of dorsally derived oligodendrocytes in the dorsal funiculus of the spinal cord 179 (Tripathi et al., 2011). Similar competition between ventrally and dorsally derived oligodendrocytes occurs in the cortex and CC in the murine forebrain (Kessaris et al., 2006). 
Although OPCs respond to neuronal electrical stimulation (Gibson et al., 2014; Li, Brus-Ramer, Martin, \& McDonald, 2010; Makinodan, Rosen, Ito, \& Corfas, 2012; Mensch et al., 2015), not all OPCs necessarily respond in the same way (discussed below) (Chittajallu, Aguirre, \& Gallo, 2004; Clarke et al., 2012; Káradóttir, Hamilton, Bakiri, \& Attwell, 2008; Spitzer et al., 2019), leading to the hypothesis that this diversity in function might be linked to developmental origin. However, there is no evidence that this is the case (Tripathi et al., 2011).

To test whether ventral OPCs can functionally compensate for the absence of dorsal OPCs, individual developmentally-distinct OPC populations in the telencephalon were ablated by region-specific expression of diphtheria toxin A (DTA). The ablation of any one of the three distinct OPC populations did not, however, cause a reduction in the total number of OLCs at P12 or in myelination in adult mice (Kessaris et al., 2006), indicating that different OLCs can functionally compensate for one another. RNA-sequencing data support these findings, as no differences in the gene expression profile between the developmentally distinct OPC populations has been detected (Marques et al., 2018). Whether ventrally and dorsally derived oligodendrocytes show transcriptional differences remains to be investigated.

Do OPCs show different propensities for self-renewal?

200

Self-renewal prevents a stem cell pool becoming depleted (stem cell exhaustion), which, in the context of OPCs, would result in an inability to generate new oligodendrocytes under homeostatic conditions and following demyelinating injury. BrdU labelling experiments had initially suggested that a non-dividing population of adult OPCs exists alongside a separate dividing population (Psachoulia, Jamen, Young, \& Richardson, 2009; Rivers et al., 2008; Simon, Götz, \& Dimou, 2011). However, a subsequent study indicated that the toxicity of BrdU in these studies may have led to erroneous conclusions being drawn on the proliferative capacity of adult OPCs (Young et al., 2013). The use of the non-toxic BrdU analogue EdU has more reliably demonstrated that all OPCS proliferate in the adult CNS (Clarke et al., 2012; Young et al., 2013). However, a difference exists between white matter (WM) and grey matter (GM) OPCs, with the former proliferating more rapidly and having a shorter cell cycle time (Dawson et al., 2003; Dimou, Simon, Kirchhoff, Takebayashi, \& Götz, 2008; Rivers et al., 2008; Young et al., 2013). This difference has been recapitulated in vitro, where WM OPCs have a 
three to four fold greater proliferative response to PDGF-AA than GM OPCs (Hill, Patel, Medved, Reiss, \& Nishiyama, 2013). WM tissue transplanted into GM areas of brain slices retain their greater proliferative response to PDGF-AA, suggesting that $\mathrm{NG}^{+}$cells in the WM have an intrinsically higher proliferative capacity than those in GM (Hill et al., 2013). The functional implication of a different proliferation, and therefore self-renewal rates, are not yet fully understood.

220

\section{Do OPCs have distinct differentiation capacities?}

Similar to the differences in proliferation, WM OPCs have a higher propensity to differentiate into mature oligodendrocytes than OPCs from GM regions (WM: 40.6\%, GM: 11\%) (Dimou et al., 2008). To resolve whether this difference is due to extrinsic or intrinsic differences between the two populations, OPCs derived from both GM and WM were transplanted into the antithetical region. Here it was shown that WM derived cells were able to differentiate more efficiently in both WM and GM than GM derived cells when transplanted into WM (Viganò, Möbius, Götz, \& Dimou, 2013). The authors posit that this demonstrates an intrinsic difference, but could not definitively rule out a role for environmental priming of the cells before transplantation.

A detailed in vivo characterisation of ion channels in neonatal OPCs identified different profiles of $\mathrm{Na}^{+}$and $\mathrm{K}^{+}$channel expression in WM and GM OPCs (Chittajallu et al., 2004; Káradóttir et al., 2008; Spitzer et al., 2019). With respect to voltage gated potassium channels, there is a marked increase in the expression of KDR (slow-inactivating delayed-rectifier) and Kir (inward-rectifier) potassium channels in GM OPCs, when compared to WM OPCs (Chittajallu et al., 2004). However, the expression of KA (fast-inactivating A-type) potassium channel is similar between the two OPC subpopulations (Chittajallu et al., 2004). The difference in potassium channel expression levels is of particular interest since oligodendrocyte specific knockout of Kir4.1 increases OPC differentiation (Schirmer et al., 2018). Therefore, and consistent with the studies discussed above, this apparent difference in the potassium channel expression between GM and WM may imply functional heterogeneity. However, these data are collected during the early postnatal period ( $p 5-10)$ and do not necessarily represent the expression profiles of adulthood. 
247 A difference in OPC expression in $\mathrm{Na}^{+}$channels has also been reported (Chittajallu et al., 2004;

248 Clarke et al., 2012; Káradóttir et al., 2008). Several studies have identified a subpopulation of 249 OPCs in both WM and GM that exhibit a transient $\mathrm{Na}_{v}$ channel mediated inward current, 250 followed by a $\mathrm{K}^{+}$channel mediated outward current, in response to depolarisation (Chittajallu 251 et al., 2004; Clarke et al., 2012; Káradóttir et al., 2008). The remaining OPCs did not show this response (Chittajallu et al., 2004; Clarke et al., 2012; Káradóttir et al., 2008). However, whether two OPC populations based on the responsiveness to depolarisation exist is still unclear, as other studies have found that all OPCs exhibit similar $\mathrm{Na}_{v}$ density and $\mathrm{Nav}$ mediated inward currents (De Biase, Nishiyama, \& Bergles, 2010; Spitzer et al., 2019). In addition, whether the ability to spike in response to depolarisation is functionally relevant for OPCs remains unknown. To date, only a positive correlation of the number of $\mathrm{Na}_{v}$ channels and active cell cycle progression of OPCs has been reported (Spitzer et al., 2019).

In addition, Spitzer and colleagues have shown that there is a higher proportion of neonatal OPCs with detectable NMDA-evoked currents in the WM, and that WM OPCs have an increased NMDA receptor density than GM OPCs (Spitzer et al., 2019). The percentage of OPCs expressing NMDA receptors decreases with age, although at different rates in WM and GM (Spitzer et al., 2019). The presence of NMDA receptors is dispensable for OPC proliferation and differentiation as the knockout of the NMDAR subunit NR1 does not show any effect on myelination (De Biase et al., 2010; Saab et al., 2016). However, the oligodendrocyte specific knockout of NMDA receptors leads to an axon pathology caused by decreased oligodendroglial axonal support in aged animals (Saab et al., 2016). Whether oligodendrocyte heterogeneity based on the capacity of metabolic support to neurons exists also remains to be investigated. In addition to the CNS region in which an OPC resides, the expression of G-protein receptor 17 (GPR17) confers OPC diversity with respect to their differentiation potential. GPR17 inhibits OPC differentiation by acting on the differentiation inhibitors ID2 and ID4 (Chen et al., 2009). GPR17-driven lineage tracing has revealed that only a proportion of adult $\mathrm{NG}^{+}$cells (75\% in the GM and 60\% in the WM) express GPR17 (Viganò et al., 2016). Using a BrdU label retention approach, it was shown that $82.0 \%$ of $\mathrm{GPR} 17^{+} / \mathrm{BrdU}^{+}$but only $23.4 \%$ of the 
GPR17-/BrdU ${ }^{+}$populations retained NG2-immunoreactivity, suggesting that more of the GPR17+ OPCs remain in cell cycle and do not undergo differentiation (Viganò et al., 2016). The block of differentiation in GPR17 $7^{+}$OPCs in homeostasis is released after various types of injuries (demyelination induced by cuprizone or EAE, and cerebral damage by acute injury or ischemia)(Coppolino et al., 2018; Viganò et al., 2016): however, how the differentiation capacity of GPR17+ OPCs compares to GPR17- OPCs after injury is not known.

\section{Are some OPCs better at regeneration than others?}

Alongside providing new oligodendrocytes for myelination during development and adulthood, OPCs have a central role in oligodendrocyte regeneration (a process known as remyelination) (Franklin \& Ffrench-Constant, 2017). In response to oligodendrocyte loss, local OPCs migrate to the site of CNS damage, proliferate, and differentiate into oligodendrocytes, or in the concomitant absence of astrocytes, into Schwann cells capable of creating new myelin sheaths (Monteiro de Castro, Deja, Ma, Zhao, \& Franklin, 2015; Zawadzka et al., 2010).

By tracing the response of dorsal OPCs to demyelination in the ventral WM of the spinal cord, it was shown that dorsal OPCs populated the lesion and differentiated in mature oligodendrocytes (Zhu et al., 2011). A subsequent study demonstrated that dorsal OPCs respond more vigorously than ventral OPCs to focal acute demyelination in the spinal cord, with more of them undergoing proliferation. Thus, following demyelination of ventral WM, where the majority of OLCs are of ventral origin, the subsequent remyelination involves a disproportionately high contribution from dorsally derived cells (Crawford, Tripathi, Richardson, \& Franklin, 2016) (Figure 2). The genetic ablation of dorsally derived OPCs led to a reduction in mature oligodendrocytes following demyelination (Crawford et al., 2016), demonstrating that ventrally derived OLCs cannot fully compensate for the lack of dorsally derived OLCs. However, the situation changes with ageing, where the majority of dorsal OLCs remains undifferentiated (presumably in an OPC state) in the aged animals, while ventral OPCs continue to differentiate into oligodendrocytes at the same rate as in young adults (Crawford et al., 2016). This suggests that the age-associated decline in OPC function has a greater impact on dorsal OPCs than on ventral OPCs. The underlying reason for this remains 
unknown. In addition, in response to the toxin-induced demyelination, dorsal OPCs show an

311 increased propensity to differentiate into Schwann cells when compared to ventral OPCS

312 (Crawford et al., 2016). However, this propensity is lost with ageing, consistent with the conclusion that dorsal and ventral OPCs age at different rates. Taken together, these data indicate that the regenerative properties of adult OPCs are determined by their developmental origin and is an example of true functional heterogeneity within the OLC lineage.

\section{Are oligodendrocytes heterogeneous in the CNS?}

The notion of oligodendrocyte diversity was first introduced by del Río Hortega who identified four different classes of oligodendrocytes based on their morphology (del Río Hortega, 1928). Class $1(\mathrm{Cl})$ oligodendrocytes occur in both WM and GM and are characterised by a high number of thin processes leading to thinly-myelinated small diameter axons. Class 2 (CII) oligodendrocytes have fewer, but thicker processes and are exclusively found in WM. Oligodendrocytes categorised in class 3 (CIII) and class 4 (CIV) are mostly found in the WM of the brain stem and spinal cord, areas with an abundance of larger diameter axons. In comparison to $\mathrm{Cl}$ and $\mathrm{Cll}$ oligodendrocytes, they are less abundant and extend fewer processes (del Río Hortega, 1928). Following this early classification of oligodendrocyte diversity, additional morphological subclasses have been identified (Murtie, Macklin, \& Corfas, 2007; Vinet et al., 2010).

The development of an MBP-GFP (membrane bound) reporter mouse line, only labelling around $1 \%$ of oligodendrocytes in the brain, has enabled imaging of the myelin sheaths formed by a single oligodendrocyte (Chong et al., 2012). 3D reconstruction revealed a diversity within the oligodendrocyte population with respect to the number of myelin sheaths formed per oligodendrocyte (between 10 and 60 myelin sheaths per oligodendrocyte) and myelin sheath length (between $20 \mu \mathrm{m}$ and $200 \mu \mathrm{m}$ per myelin sheath) (Chong et al., 2012). This diversity is not region-specific, and occurs along axons with similar functional properties (Chong et al., 2012; Tomassy et al., 2014), suggesting that internode length might not be determined by the regional diversity of oligodendrocytes (as proposed by del Rio Hortega), but rather local environmental cues. Indeed, using an in vitro co-culture of cortical OPCs with 
neurons, Chong and colleagues were able to demonstrate that the density of OPCs (not

343 oligodendrocytes) negatively regulates the myelinogenic potential of oligodendrocytes

344 through repulsive interaction (Chong et al., 2012). Whether there is a difference in OPC density in different CNS regions and how the local density of OPCs would be regulated in the CNS to explain the observed morphological subclasses of oligodendrocytes remains unknown.

To assess the intrinsic diversity in regional OLC populations without the influence of axon properties, Bechler and colleagues have examined the compact myelin sheath formation of cortical and spinal cord OPCs in an assay where artificial microfibres substitute for the role of the axon in providing a substrate for myelination. Oligodendrocytes from the spinal cord formed myelin sheaths which are twice as long as those formed by cortical oligodendrocytes, even though the number of sheaths formed per oligodendrocyte was similar (Bechler, Byrne, \& Ffrench-Constant, 2015). This suggests that the origin of the OPCs determines the myelinogenic potential of the oligodendrocytes. However, the difference in internode length formed by cortical and spinal cord oligodendrocytes was less pronounced when the OPCs of different origins were cultured on dorsal root ganglion neurons or brain slices, indicating that neurons also influence the myelinogenic potential of the oligodendrocytes (Bechler et al., 2015).

The optimisation of the single-cell RNA sequencing of CNS cells has allowed the analysis of oligodendrocyte diversity to be explored in greater depth. OLCs in ten different CNS regions of juvenile and adult mouse CNS revealed 12 distinct OLC populations spanning the differentiation stages of OPCs to mature oligodendrocytes. In the juvenile mouse, all CNS regions contain oligodendrocytes from at least 2 different oligodendrocyte populations. Whereas one mature oligodendrocyte population was present in all CNS regions, the other oligodendrocyte populations are prevalent in certain CNS regions. However, within the adult brain regions examined (cortex and CC) the diversity of oligodendrocyte populations is reduced, with only two oligodendrocyte populations being present (Marques et al., 2016). Whether the transcriptionally different oligodendrocyte populations fulfil distinct functions in the brain remains to be investigated. These findings raise several important questions including, how can transcriptional diversity of oligodendrocytes arise from transcriptionally homogenous OPCs (Marques et al., 2018)? Possible explanations include technical limitations 
374 of the sequencing technique to study gene expression in OPC (limited amounts of RNA, 375 fragility of OPC population) or environmental influences exerted during, or after, the 376 oligodendrocyte differentiation process.

378 Strong evidence for functional heterogeneity of oligodendrocytes has been obtained using 379 three different viruses to label oligodendrocytes, together with neuronal axon projections of motor and sensory neurons in the CC. The analysis revealed that collosal oligodendrocytes can be classified into three categories: those that either preferentially myelinate axons from 1) the motor cortex, 2) the sensory cortex, and 3) from both brain regions without preference ( $75 \%$ of all oligodendrocytes assessed) (Osanai et al., 2017). It is conceivable that the $25 \%$ of oligodendrocytes showing a preference towards specific axons are adult-born oligodendrocytes, specifically myelinating an axon based on its activity.

\section{Concluding remarks}

An expanding body of evidence has been published describing phenotypical differences within the OPC and oligdendrocyte populations (Table 1). However, only a minortiy of these publications addresses the important question of whether the observed phenotypical differences are intrinsically driven (indicating OLC heterogeneity) or dictated by environmental cues (OLC functional plasticity). As intrinsic heterogeneity is often established due to different extrinsic (developmental) signals, the definition of intrinsic and extrinsic heterogeneity can be blurred. The definition implies that extrinsically heterogeneous cells would show similar properties within an identical environment. In contrast, cells that are intrinsically heterogeneous will still exhibit different functional behaviour even in the same environment. While one study argues for a non-existence of oligodendrocyte diversity (Chong et al., 2012), other studies showed intrinsic diversity of aspects of OPC, such as OPC differentiation capacity, (Crawford et al., 2016; Viganò et al., 2013) and oligodendrocyte biology (Bechler et al., 2015). However, whether these intrinsic differences have any functional implications has only been adressed in one study (Table 1). Crawford and colleagues showed that dorsal OPCs are the proportionally greater contributors to WM remyelination, and that the deletion of dorsal OPCs leads to a reduced remyelination effeciency (Crawford et al., 2016) (Table 1). Nevertheless, no evidence has been found for the 
406 functional heterogeneity in the homeostatic adult CNS, leaving the field without the definitive

407 proof required to unambiguously assert heterogeneity. However, the discovery of new

408 functions of OLCs are likely to reveal other examples of functional heterogeneity, and allow

409 current phenotypic descriptions of diversity to be better mapped on to newly elucidated OLCs

410 functions.

411

412 In favour of the existence of functional OLCs heterogeneity is the notion that the cortex, an

413 area coordinating complex tasks, is mainly populated by dorsal OLCs, whereas other

414 evolutionarily conserved brain areas are populated by ventral OLCs, suggesting that a variety

415 of oligodendrocyte subtypes are needed for optimal CNS function. In addition, the most

416 heterogeneous set of myelination profiles of the murine cerebral cortex exists in the upper

417 layers which is due to neurons from different cortical layers having different longitudinal

418 myelination profiles along their axons (Tomassy et al., 2014). While this effect might be driven

419 by neuronal activity, it is possible that distinct oligodendrocyte subpopulations are needed to

420 create such a specific myelination pattern. To this end, oligodendrocytes are transcriptionally

421 distinct in the adult CNS, which is indicative of functional distinct oligodendrocyte

422 subpopulations (Marques et al., 2016). This would echo what is know about the other

423 principal macroglial cell type, the astrocyte, where it has been shown that functionally distinct

424 astrocyte populations are necessary to support optimal neuronal transmission (Tsai et al.,

425 2012). As oligodendrocytes are also critical for neuron circuit function, it is likely that distinct

426 oligodendrocytes exist to meet the special needs of different neuronal circuits. Furthermore,

427 OPCs and oligodendrocytes form intercellular connections with neurons (via synapses) and

428 astrocytes (via gap junctions), respectively. Neurons exhibit functional heterogeneity with

429 respect to their mode of transmission and firing patterns, and astrocytes were shown to 430 become specialised for interactions with their own particular neuronal neighbours (Tsai et al.,

431 2012). Therefore, the existence of OLCs heterogeneity to accommodate the specific

432 functional requirements of individual neuron-glia networks is likely. 
Bechler, M. E., Byrne, L., \& ffrench-Constant, C. (2015). CNS Myelin Sheath Lengths Are an Intrinsic Property of Oligodendrocytes. Current Biology: CB, 25(18), 2411-2416. http://dx.doi.org/10.1016/j.cub.2015.07.056

Cahoy, J. D., Emery, B., Kaushal, A., Foo, L. C., Zamanian, J. L., Christopherson, K. S., et al. (2008). A transcriptome database for astrocytes, neurons, and oligodendrocytes: a new resource for understanding brain development and function. The Journal of Neuroscience: the Official Journal of the Society for Neuroscience, 28(1), 264-278. http://doi.org/10.1523/JNEUROSCI.4178-07.2008

Cai, J., Qi, Y., Hu, X., Tan, M., Liu, Z., Zhang, J., et al. (2005). Generation of oligodendrocyte precursor cells from mouse dorsal spinal cord independent of Nkx6 regulation and Shh signaling. Neuron, 45(1), 41-53. http://doi.org/10.1016/j.neuron.2004.12.028

Chandran, S., Kato, H., Gerreli, D., Compston, A., Svendsen, C. N., \& Allen, N. D. (2003). FGFdependent generation of oligodendrocytes by a hedgehog-independent pathway.

Development (Cambridge, England), 130(26), 6599-6609.

http://doi.org/10.1242/dev.00871

Chen, Y., Wu, H., Wang, S., Koito, H., Li, J., Ye, F., et al. (2009). The oligodendrocyte-specific $\mathrm{G}$ protein-coupled receptor GPR17 is a cell-intrinsic timer of myelination. Nature Neuroscience, 12(11), 1398-1406. http://doi.org/10.1038/nn.2410

Chittajallu, R., Aguirre, A., \& Gallo, V. (2004). NG2-positive cells in the mouse white and grey matter display distinct physiological properties. The Journal of Physiology, 561(Pt 1), 109-122. http://doi.org/10.1113/jphysiol.2004.074252

Chong, S. Y. C., Rosenberg, S. S., Fancy, S. P. J., Zhao, C., Shen, Y.-A. A., Hahn, A. T., et al. (2012). Neurite outgrowth inhibitor Nogo-A establishes spatial segregation and extent of oligodendrocyte myelination. Proceedings of the National Academy of Sciences of the United States of America, 109(4), 1299-1304. http://doi.org/10.1073/pnas.1113540109

Clarke, L. E., Young, K. M., Hamilton, N. B., Li, H., Richardson, W. D., \& Attwell, D. (2012). Properties and fate of oligodendrocyte progenitor cells in the corpus callosum, motor cortex, and piriform cortex of the mouse. The Journal of Neuroscience : the Official Journal of the Society for Neuroscience, 32(24), 8173-8185. http://doi.org/10.1523/JNEUROSCI.0928-12.2012

Coppolino, G. T., Marangon, D., Negri, C., Menichetti, G., Fumagalli, M., Gelosa, P., et al. (2018). Differential local tissue permissiveness influences the final fate of GPR17expressing oligodendrocyte precursors in two distinct models of demyelination. Glia, 66(5), 1118-1130. http://doi.org/10.1002/glia.23305 
Susceptibility to Age-Associated Functional Decline. Cell Reports, 15(4), 761-773. http://doi.org/10.1016/j.celrep.2016.03.069

Dawson, M. R. L., Polito, A., Levine, J. M., \& Reynolds, R. (2003). NG2-expressing glial progenitor cells: an abundant and widespread population of cycling cells in the adult rat CNS. Molecular and Cellular Neurosciences, 24(2), 476-488. https://doi.org/10.1016/S1044-7431(03)00210-0

De Biase, L. M., Nishiyama, A., \& Bergles, D. E. (2010). Excitability and synaptic communication within the oligodendrocyte lineage. The Journal of Neuroscience: the Official Journal of the Society for Neuroscience, 30(10), 3600-3611. https://doi.org/10.1523/JNEUROSCI.6000-09.2010

del Río Hortega, P. (1928). Tercera aportación al conocimiento morfológico e interpretación funcional de la oligodendroglía. Memorias De La Real Sociedad Española De Historia Natural, XIV, 5-122.

Dimou, L., Simon, C., Kirchhoff, F., Takebayashi, H., \& Götz, M. (2008). Progeny of Olig2expressing progenitors in the gray and white matter of the adult mouse cerebral cortex. The Journal of Neuroscience: the Official Journal of the Society for Neuroscience, 28(41), 10434-10442. http://doi.org/10.1523/JNEUROSCI.2831-08.2008

Fogarty, M., Richardson, W. D., \& Kessaris, N. (2005). A subset of oligodendrocytes generated from radial glia in the dorsal spinal cord. Development (Cambridge, England), 132(8), 1951-1959. http://doi.org/10.1242/dev.01777

Franklin, R. J. M., \& ffrench-Constant, C. (2017). Regenerating CNS myelin - from mechanisms to experimental medicines. Nature Reviews. Neuroscience, 18(12), 753769. http://doi.org/10.1038/nrn.2017.136

Gibson, E. M., Purger, D., Mount, C. W., Goldstein, A. K., Lin, G. L., Wood, L. S., et al. (2014). Neuronal activity promotes oligodendrogenesis and adaptive myelination in the mammalian brain. Science (New York, N.Y.), 344(6183), 1252304-1252304. http://doi.org/10.1126/science.1252304

Hill, R. A., Patel, K. D., Medved, J., Reiss, A. M., \& Nishiyama, A. (2013). NG2 cells in white matter but not gray matter proliferate in response to PDGF. The Journal of Neuroscience : the Official Journal of the Society for Neuroscience, 33(36), 14558-14566. http://doi.org/10.1523/JNEUROSCI.2001-12.2013

Kang, S. H., Fukaya, M., Yang, J. K., Rothstein, J. D., \& Bergles, D. E. (2010). NG2+ CNS glial progenitors remain committed to the oligodendrocyte lineage in postnatal life and following neurodegeneration. Neuron, 68(4), 668-681. http://doi.org/10.1016/j.neuron.2010.09.009 
Karram, K., Goebbels, S., Schwab, M., Jennissen, K., Seifert, G., Steinhäuser, C., et al. (2008). NG2-expressing cells in the nervous system revealed by the NG2-EYFP-knockin mouse. Genesis (New York, N.Y. : 2000), 46(12), 743-757. http://doi.org/10.1002/dvg.20440

Káradóttir, R., Hamilton, N. B., Bakiri, Y., \& Attwell, D. (2008). Spiking and nonspiking classes of oligodendrocyte precursor glia in CNS white matter. Nature Neuroscience, 11(4), 450-456. http://doi.org/10.1038/nn2060

Kessaris, N., Fogarty, M., lannarelli, P., Grist, M., Wegner, M., \& Richardson, W. D. (2006). Competing waves of oligodendrocytes in the forebrain and postnatal elimination of an embryonic lineage. Nature Neuroscience, 9(2), 173-179. http://doi.org/10.1038/nn1620

Kuhlbrodt, K., Herbarth, B., Sock, E., Hermans-Borgmeyer, I., \& Wegner, M. (1998). Sox10, a novel transcriptional modulator in glial cells. Journal of Neuroscience, 18(1), 237-250. https://doi.org/10.1523/JNEUROSCI.18-01-00237

Langseth, A. J., Munji, R. N., Choe, Y., Huynh, T., Pozniak, C. D., \& Pleasure, S. J. (2010). Wnts influence the timing and efficiency of oligodendrocyte precursor cell generation in the telencephalon. The Journal of Neuroscience : the Official Journal of the Society for Neuroscience, 30(40), 13367-13372. http://doi.org/10.1523/JNEUROSCI.1934-10.2010

Li, Q., Brus-Ramer, M., Martin, J. H., \& McDonald, J. W. (2010). Electrical stimulation of the medullary pyramid promotes proliferation and differentiation of oligodendrocyte progenitor cells in the corticospinal tract of the adult rat. Neuroscience Letters, 479(2), 128-133. http://doi.org/10.1016/j.neulet.2010.05.043

Ligon, K. L., Kesari, S., Kitada, M., Sun, T., Arnett, H. A., Alberta, J. A., et al. (2006). Development of NG2 neural progenitor cells requires Olig gene function. Proceedings of the National Academy of Sciences of the United States of America, 103(20), 7853-7858. http://doi.org/10.1073/pnas.0511001103

Linnington, C., Webb, M., \& Woodhams, P. L. (1984). A novel myelin-associated glycoprotein defined by a mouse monoclonal antibody. Journal of Neuroimmunology, 6(6), 387-396. https://doi.org/10.1016/0165-5728(84)90064-X

Makinodan, M., Rosen, K. M., Ito, S., \& Corfas, G. (2012). A critical period for social experience-dependent oligodendrocyte maturation and myelination. Science (New York, N.Y.), 337(6100), 1357-1360. http://doi.org/10.1126/science.1220845

Marques, S., van Bruggen, D., Vanichkina, D. P., Floriddia, E. M., Munguba, H., Väremo, L., et al. (2018). Transcriptional Convergence of Oligodendrocyte Lineage Progenitors during Development. Developmental Cell, 46(4), 504-517.e7. http://doi.org/10.1016/j.devcel.2018.07.005

Marques, S., Zeisel, A., Codeluppi, S., van Bruggen, D., Mendanha Falcão, A., Xiao, L., et al. (2016). Oligodendrocyte heterogeneity in the mouse juvenile and adult central nervous 
system. Science (New York, N.Y.), 352(6291), 1326-1329.

http://doi.org/10.1126/science.aaf6463

Mensch, S., Baraban, M., Almeida, R., Czopka, T., Ausborn, J., Manira, El, A., \& Lyons, D. A. (2015). Synaptic vesicle release regulates myelin sheath number of individual oligodendrocytes in vivo. Nature Neuroscience, 18(5), 628-630. http://doi.org/10.1038/nn.3991

Monteiro de Castro, G., Deja, N. A., Ma, D., Zhao, C., \& Franklin, R. J. M. (2015). Astrocyte Activation via Stat3 Signaling Determines the Balance of Oligodendrocyte versus Schwann Cell Remyelination. The American Journal of Pathology, 185(9), 2431-2440. http://doi.org/10.1016/j.ajpath.2015.05.011

Moyon, S., Dubessy, A. L., Aigrot, M.-S., Trotter, M., Huang, J. K., Dauphinot, L., et al. (2015). Demyelination causes adult CNS progenitors to revert to an immature state and express immune cues that support their migration. The Journal of Neuroscience: the Official Journal of the Society for Neuroscience, 35(1), 4-20. http://doi.org/10.1523/JNEUROSCI.0849-14.2015

Murtie, J. C., Macklin, W. B., \& Corfas, G. (2007). Morphometric analysis of oligodendrocytes in the adult mouse frontal cortex. Journal of Neuroscience Research, 85(10), 2080-2086. http://doi.org/10.1002/jnr.21339

Osanai, Y., Shimizu, T., Mori, T., Yoshimura, Y., Hatanaka, N., Nambu, A., et al. (2017). Rabies virus-mediated oligodendrocyte labeling reveals a single oligodendrocyte myelinates axons from distinct brain regions. Glia, 65(1), 93-105. http://doi.org/10.1002/glia.23076

Poduslo, S. E., \& Norton, W. T. (1972). Isolation and some chemical properties of oligodendroglia from calf brain. Journal of Neurochemistry, 19(3), 727-736. https://doi.org/10.1111/j.1471-4159.1972.tb01388.x

Pringle, N. P., \& Richardson, W. D. (1993). A singularity of PDGF alpha-receptor expression in the dorsoventral axis of the neural tube may define the origin of the oligodendrocyte lineage. Development (Cambridge, England), 117(2), 525-533.

Pringle, N. P., Mudhar, H. S., Collarini, E. J., \& Richardson, W. D. (1992). PDGF receptors in the rat CNS: during late neurogenesis, PDGF alpha-receptor expression appears to be restricted to glial cells of the oligodendrocyte lineage. Development (Cambridge, England), 115(2), 535-551.

Psachoulia, K., Jamen, F., Young, K. M., \& Richardson, W. D. (2009). Cell cycle dynamics of NG2 cells in the postnatal and ageing brain. Neuron Glia Biology, 5(3-4), 57-67. http://doi.org/10.1017/S1740925X09990354

Raff, M. C., Miller, R. H., \& Noble, M. (1983). A glial progenitor cell that develops in vitro into an astrocyte or an oligodendrocyte depending on culture medium. Nature, 303(5916), 390-396. http://doi.org/10.1038/303390a0 
Rivers, L. E., Young, K. M., Rizzi, M., Jamen, F., Psachoulia, K., Wade, A., et al. (2008). PDGFRA/NG2 glia generate myelinating oligodendrocytes and piriform projection neurons in adult mice. Nature Neuroscience, 11(12), 1392-1401. http://doi.org/10.1038/nn.2220

Saab, A. S., Tzvetavona, I. D., Trevisiol, A., Baltan, S., Dibaj, P., Kusch, K., et al. (2016). Oligodendroglial NMDA Receptors Regulate Glucose Import and Axonal Energy Metabolism. Neuron, 91(1), 119-132. http://doi.org/10.1016/j.neuron.2016.05.016

Schirmer, L., Möbius, W., Zhao, C., Cruz-Herranz, A., Ben Haim, L., Cordano, C., et al. (2018). Oligodendrocyte-encoded Kir4.1 function is required for axonal integrity. eLife, 7. http://doi.org/10.7554/eLife.36428

Simon, C., Götz, M., \& Dimou, L. (2011). Progenitors in the adult cerebral cortex: cell cycle properties and regulation by physiological stimuli and injury. Glia, 59(6), 869-881. http://doi.org/10.1002/glia.21156

Sobel, R. A., Greer, J. M., Isaac, J., Fondren, G., \& Lees, M. B. (1994). Immunolocalization of proteolipid protein peptide 103-116 in myelin. Journal of Neuroscience Research, 37(1), 36-43. http://doi.org/10.1002/jnr.490370106

Sommer, I., \& Schachner, M. (1981). Monoclonal antibodies (O1 to O4) to oligodendrocyte cell surfaces: an immunocytological study in the central nervous system. Developmental Biology, 83(2), 311-327. https://doi.org/10.1016/0012-1606(81)90477-2

Spitzer, S. O., Sitnikov, S., Kamen, Y., Evans, K. A., Kronenberg-Versteeg, D., Dietmann, S., et al. (2019). Oligodendrocyte Progenitor Cells Become Regionally Diverse and Heterogeneous with Age. Neuron. http://doi.org/10.1016/j.neuron.2018.12.020

Stallcup, W. B., \& Beasley, L. (1987). Bipotential glial precursor cells of the optic nerve express the NG2 proteoglycan. Journal of Neuroscience, 7(9), 2737-2744. https://doi.org/10.1523/JNEUROSCI.07-09-02737

Sternberger, N. H., Itoyama, Y., Kies, M. W., \& Webster, H. D. (1978). Myelin basic protein demonstrated immunocytochemically in oligodendroglia prior to myelin sheath formation. Proceedings of the National Academy of Sciences of the United States of America, 75(5), 2521-2524. http://doi.org/10.1073/pnas.75.5.2521

Sternberger, N. H., Quarles, R. H., Itoyama, Y., \& Webster, H. D. (1979). Myelin-associated glycoprotein demonstrated immunocytochemically in myelin and myelin-forming cells of developing rat. Proceedings of the National Academy of Sciences of the United States of America, 76(3), 1510-1514. https://doi.org/10.1073/pnas.76.3.1510

Tomassy, G. S., Berger, D. R., Chen, H.-H., Kasthuri, N., Hayworth, K. J., Vercelli, A., et al. (2014). Distinct profiles of myelin distribution along single axons of pyramidal neurons 
Tripathi, R. B., Clarke, L. E., Burzomato, V., Kessaris, N., Anderson, P. N., Attwell, D., \& Richardson, W. D. (2011). Dorsally and ventrally derived oligodendrocytes have similar electrical properties but myelinate preferred tracts. The Journal of Neuroscience : the Official Journal of the Society for Neuroscience, 31(18), 6809-6819. http://doi.org/10.1523/JNEUROSCI.6474-10.2011

Tsai, H.-H., Li, H., Fuentealba, L. C., Molofsky, A. V., Taveira-Marques, R., Zhuang, H., et al. (2012). Regional astrocyte allocation regulates CNS synaptogenesis and repair. Science (New York, N.Y.), 337(6092), 358-362. http://doi.org/10.1126/science.1222381

Vallstedt, A., Klos, J. M., \& Ericson, J. (2005). Multiple dorsoventral origins of oligodendrocyte generation in the spinal cord and hindbrain. Neuron, 45(1), 55-67. http://doi.org/10.1016/j.neuron.2004.12.026

Viganò, F., Möbius, W., Götz, M., \& Dimou, L. (2013). Transplantation reveals regional differences in oligodendrocyte differentiation in the adult brain. Nature Neuroscience, 16(10), 1370-1372. http://doi.org/10.1038/nn.3503

Viganò, F., Schneider, S., Cimino, M., Bonfanti, E., Gelosa, P., Sironi, L., et al. (2016). GPR17 expressing NG2-Glia: Oligodendrocyte progenitors serving as a reserve pool after injury. Glia, 64(2), 287-299. http://doi.org/10.1002/glia.22929

Vinet, J., Lemieux, P., Tamburri, A., Tiesinga, P., Scafidi, J., Gallo, V., \& Sík, A. (2010). Subclasses of oligodendrocytes populate the mouse hippocampus. The European Journal of Neuroscience, 31(3), 425-438. http://doi.org/10.1111/j.14609568.2010.07082.x

Xiao, L., Ohayon, D., McKenzie, I. A., Sinclair-Wilson, A., Wright, J. L., Fudge, A. D., et al. (2016). Rapid production of new oligodendrocytes is required in the earliest stages of motor-skill learning. Nature Neuroscience, 19(9), 1210-1217. http://doi.org/10.1038/nn.4351

Young, K. M., Psachoulia, K., Tripathi, R. B., Dunn, S.-J., Cossell, L., Attwell, D., et al. (2013). Oligodendrocyte dynamics in the healthy adult CNS: evidence for myelin remodeling. Neuron, 77(5), 873-885. http://doi.org/10.1016/j.neuron.2013.01.006

Zawadzka, M., Rivers, L. E., Fancy, S. P. J., Zhao, C., Tripathi, R., Jamen, F., et al. (2010). CNSresident glial progenitor/stem cells produce Schwann cells as well as oligodendrocytes during repair of CNS demyelination. Cell Stem Cell, 6(6), 578-590. http://doi.org/10.1016/j.stem.2010.04.002

Zhou, Q., Wang, S., \& Anderson, D. J. (2000). Identification of a novel family of oligodendrocyte lineage-specific basic helix-loop-helix transcription factors. Neuron, 25(2), 331-343. https://doi.org/10.1016/S0896-6273(00)80898-3 
710 Zhu, Q., Whittemore, S. R., Devries, W. H., Zhao, X., Kuypers, N. J., \& Qiu, M. (2011). Dorsally-derived oligodendrocytes in the spinal cord contribute to axonal myelination during development and remyelination following focal demyelination. Glia, 59(11), 1612-1621. http://doi.org/10.1002/glia.21203 
Figure legends

Figure 1: Overlap of OPC markers

716

717 Overlap of OPC markers (NG2, PDGFRA and A2B5) and oligodendrocyte lineage cell markers

718 (Olig2 and Sox10) in neonatal (left) and adult (right) OPCs based on published in vivo lineage 719 tracing experiments (Clarke et al., 2012; Kang et al., 2010; Karram et al., 2008; Ligon et al., 720 2006; Rivers et al., 2008; Stallcup \& Beasley, 1987). A2B5 data was generated from immunostaining of with the A2B5 antibody (unpublished data). The overlap of OPC marker expression changes during development: adult OPCs show a higher overlap of the OPC marker proteins when compared to neonatal OPCs.

724

Figure 2: Developmental origin of OPCs determines their remyelination response

727 Following a focal toxin-induced demyelination injury dorsal OPCs make a disproportionately high contribution to remyelination when compared to ventral OPCs. Detailed analysis of the OPC response to the injury showed that a higher proliferative response of dorsal OPCs causes their increased response to demyelination. $\mathrm{MGE}=$ medial ganglionic eminence, $\mathrm{AEP}=$ anterior entopeduncular, LGE = lateral ganglionic eminence, $\mathrm{CGE}=$ caudal ganglionic eminence, $\mathrm{CC}=$ corpus callosum, $\mathrm{AC}=$ Anterior commisure, $\mathrm{p}=$ progenitor domain, $\mathrm{MN}=$ motor neuron, $\mathrm{dP}=$ dorsal progenitor domain, $D F=$ dorsal funiculus, $L F=$ lateral funiculus.

Table legends

736

Table 1: Summary of current literature on OPC and oligodendrocyte diversity

Several phenotypical differences have been described between subclasses of oligodendrocyte lineage cells. However, the assessment of phenotypic differences does not allow to distinguish between cell/lineage plasticity and heterogeneity. Therefore, functional differences between subclasses of oligodendrocyte lineage cells need to be investigated to unambiguously prove heterogeneity. 